\title{
The Structure of the Multi-Phase ISM in Low Surface Brightness Galaxies
}

\author{
M. Spaans ${ }^{1}$ \\ Harvard-Smithsonian Center for Astrophysics, 60 Garden Street, \\ Cambridge, MA 02138
}

\begin{abstract}
The multi-phase structure of the interstellar medium in low surface brightness galaxies is investigated and compared to observations. It is found that the ambient pressure and metallicity very strongly influence the abundances of molecular hydrogen and carbon monoxide. The emissivity of the latter is computed and found to agree naturally with the upper limits measured for low surface brightness galaxies. The implications for star formation efficiency and galaxy evolution are discussed.
\end{abstract}

\section{Introduction}

Low surface brightness (LSB) disk galaxies represent a class of galactic systems which have experienced very slow evolution since their formation epoch. Their low surface brightnesses $\left(<1 \mathrm{mag} / \operatorname{arcsec}^{2}\right.$ below the canonical Freeman $(1970)$ value of $\mu_{0}^{B}=21.65 \pm 0.3 \mathrm{mag} / \mathrm{arcsec}^{2}$ ) indicate that, over the age of the Universe, their mean stellar birthrate per unit area has been significantly lower than that of typical high surface brightness (HSB) disks. Their current rate of star formation is similarly low - while some HII regions do exist in LSBs, the global star formation rate in LSBs is lower by an order of magnitude than comparably sized HSBs (McGaugh 1992). The lack of significant star formation is reflected in the low metallicities of LSBs, which are typically $<1 / 3$ solar (McGaugh 1992). Not coincidentally, LSBs are also very gas-rich systems - McGaugh \& de Blok (1997) found that the gas mass fraction of galaxy disks correlates strongly with surface brightness, such that in LSBs, as much as $50 \%$ of the disk mass is in the form of gas, compared to $\sim 10 \%$ at high surface brightnesses.

The suppressed rate of star formation in LSB disks must ultimately be connected to the differing physical conditions of the ISM between LSB and HSB disk galaxies. As star formation is presumed to take place in molecular clouds, the molecular content of LSBs is of particular interest. In typical HSB spirals, the mass of molecular gas is comparable to that in neutral HI (e.g., Young \& Knezek 1989). The situation in LSBs may be quite different -- while several CO surveys of LSBs have been made (e.g., Schombert et al. 1990 (S90)), CO emission has not been detected in any LSB disk galaxy. If CO emission traces molecular gas content in the same way as in normal HSB galaxies, then the

${ }^{1}$ Hubble Fellow 
upper limits on molecular gas in LSBs are $M_{\mathrm{H}_{2}} / M_{\mathrm{HI}}<0.1$. These upper limits have led to the speculation that the low disk surface densities in LSBs preclude molecular cloud formation and, in turn, inhibit star formation (e.g., S90; van der Hulst et al. 1993; Bothun et al. 1997). Alternatively, the lack of CO detection may simply reflect the fact that the $\mathrm{CO} / \mathrm{H}_{2}$ conversion factor is not a universal constant, so that perhaps large quantities of $\mathrm{H}_{2}$ exist despite the lack of detected $\mathrm{CO}$ emission.

Unfortunately, an observational answer to the question of the molecular content of LSBs is inexorably tied to the $\mathrm{CO} / \mathrm{H}_{2}$ conversion factor and its dependency on environment. For example, Wilson (1995) and Israel (1997) recently showed that the $\mathrm{CO} / \mathrm{H}_{2}$ conversion factor is a strong function of metallicity; this dependency raises the upper limits on the derived molecular content of LSBs. Nonetheless, even accounting for metallicity effects, previous CO surveys should have detected $\mathrm{CO}$ in LSBs if they had $M_{\mathrm{H}_{2}} / M_{\mathrm{HI}}$ ratios similar to HSBs. Unfortunately other dependencies also play a role, such as the local gas density and temperature (e.g., Maloney \& Black 1988, Scoville \& Sanders 1987), which in turn are affected by the ionizing radiation field and density structure ("clumpiness") of the ISM. In LSBs all these factors may well be significantly different than expected for HSBs.

To explore the ISM properties of LSB galaxies in a manner independent of the $\mathrm{CO} / \mathrm{H}_{2}$ conversion factor, we take a complementary, theoretical route towards understanding the molecular content of LSB galaxies. We construct models of an inhomogeneous ISM under varying physical conditions, spanning a range of disk galaxy types. The models employ a Monte Carlo approach to radiative transfer (see Spaans 1996), and explicitly solve for the CO emissivity and $M_{\mathrm{H}_{2}} / M_{\mathrm{HI}}$ ratio in galactic disks. We investigate models on a grid of metallicity, surface brightness, and ISM density structure, tracking the changing physical conditions between LSB and HSB disk galaxies.

\section{Model Description}

The code developed by Spaans (1996) and its extensions as discussed in Mihos, Spaans \& McGaugh (1998) is used to derive the physical and chemical structure of the ambient ISM in LSBs. The interested reader is referred to these papers for a detailed description of the code's structure. The main features can be summarized as follows.

1) For a given metallicity, geometry, global pressure structure and distribution of illuminating (ultraviolet) sources, the thermal and chemical balance of the medium is computed in three dimensions. The continuum (dust attenuation) and line transfer is modeled through a Monte Carlo method. The self-shielding of $\mathrm{H}_{2}$ and $\mathrm{CO}$ and the shielding of $\mathrm{CO}$ by $\mathrm{H}_{2}$ absorption lines is explicitly included. The heating processes include photo-electric emission by dust grains, cosmic ray heating, collisional de-excitation of ultraviolet pumped $\mathrm{H}_{2}$, and $\mathrm{H}_{2}$ dissociation heating. The cooling processes include fine-structure emission of $\mathrm{C}^{+}, \mathrm{C}$ and $\mathrm{O}$, rotational line emission of $\mathrm{CO}$, and vibrational $(v=1-0) \mathrm{H}_{2}$ emission.

2) The solutions to the thermal balance equations allow, for a given hydrodynamic pressure and metallicity, multiple solutions (Norman \& Spaans 1997). 
These constitute the possible multi-phase structure of the ISM as first suggested by Field, Goldsmith, \& Habing (1969). If multiple solutions exist, then one finds from a stability analysis that there is a $\sim 10^{4} \mathrm{~K}$ diffuse medium and a $\sim 50 \mathrm{~K}$ dense component. It is the density structure derived from these solutions which couples strongly with the chemical balance of interstellar gas, and therefore with the amount of molecular gas which is supported by the stellar radiation field and the ambient pressure of the galaxy. The cold component has a typical density of $\sim 50-300 \mathrm{~cm}^{-3}$ and is representative of diffuse and translucent clouds in the Milky Way.

\subsection{Model Parameters and Their Implementation}

To investigate the molecular content of the ISM the following model parameters are considered: average gas density, the average interstellar radiation field (ISRF) in the LSB, metallicity, surface density, and ISM density structure. These parameters are not all independent. To capture the essential dependencies of the ISM structure on ambient physical conditions the following scaling relations are adopted. The HI volume density $n_{\mathrm{HI}}$ correlates with HI surface density $\Sigma_{\mathrm{HI}}$ according to

$$
n_{\mathrm{HI}}=\Sigma_{\mathrm{HI}} / H
$$

where $H=300 \mathrm{pc}$ is the scale height of the galaxy model. Using data from de Blok et al. (1996), one can derive a rough correlation between local surface brightness $\mu^{B}$ and local HI density:

$$
\log \Sigma_{\mathrm{HI}} \approx-0.12 * \mu^{B}+3.6
$$

This relationship shows that $\mathrm{HI}$ surface density and stellar surface brightness do not drop off in lockstep; instead, the HI surface density falls off more slowly. As surface brightness decreases, the gas mass fraction of the disk increases, such that very low surface brightness disks $\left(\mu_{0}^{B}>23\right)$ have fully half their baryonic mass in the form of gas.

If one relates the surface brightness and radius $r$ as

$$
\mu^{B}=\mu_{0}^{B}+1.086 *(r / h),
$$

with $\mu_{0}^{B}$ the central surface brightness in B mags per square arcsecond one finds

$$
\log \Sigma_{\mathrm{HI}} \approx-0.12 * \mu_{0}^{B}-0.13 *(r / h)+3.6
$$

Again, the relationship implies that, as a function of radius, the HI surface density drops off more slowly than the stellar surface brightness, reproducing the extended gaseous disks observed in disk galaxies. In this parametrization, the gas surface density is exponential, but with a scale length 3.3 times larger than that for the stars. See Mihos et al. (1998) for further details.

To parameterize the strength of the ISRF in our models, we assume that the ISRF is dominated by the contribution from the stellar populations in galaxies. Under this assumption, the ISRF scales with surface brightness:

$$
I_{\mathrm{UV}}=I_{\mathrm{UV}}(M W) * 10^{0.4 *\left(\mu_{0}^{B}(\mathrm{MW})-\mu_{0}^{B}\right)}
$$


where $I_{\mathrm{UV}}(\mathrm{MW})$ is the strength of the ISRF in the Milky Way given by Draine (1978), and $\mu_{0}^{B}(\mathrm{MW})$ is the central surface brightness of the Milky Way disk (assumed to be $21 \mathrm{mag}$ arcsec${ }^{-2}$ ). Furthermore, we assume that the spectral shape is independent of surface brightness, or, equivalently, that the stellar populations which give rise to the ISRF do not drastically change as a function of surface brightness.

Finally, we need to characterize the inhomogeneity of the dense phase, if it is supported, in the models. This inhomogeneity can be parameterized by choosing a certain volume fraction $F$ of the gas in high density clumps with a fixed density contrast $R$. The size of the clumps is not varied and assumed equal to $2 \mathrm{pc}$, typical for translucent clouds in the Milky Way. By investigating a range of density contrasts, and therefore clump extinction, this somewhat arbitrary length does not strongly influence the results. We calculate one model, " $\mathrm{H}$ ", which is completely homogeneous and lacks any density structure. Two more models are explored which have modest amounts of structure ("I1,I2", with $R=2,4$ and $F=0.5,0.25$; see Mihos et al. 1998). Finally, the clumpy ISM models ("C1,C2", $R=20,60$ and $F=0.25,0.1$; see Mihos et al. 1998) are chosen to represent our own Galaxy at high ISM pressure.

With these parameterizations, we are left with four variables describing the model galaxies: disk surface brightness, metallicity, pressure, and clumpiness. We create a grid of models spanning a range of plausible values: central surface brightness $\mu_{0}^{B}=21 \rightarrow 24$, metallicity $Z / Z_{\odot}=1 \rightarrow 0.1$, and ISM types $\mathrm{H}$ (homogeneous, $P \sim 10^{3} \mathrm{~K} \mathrm{~cm}^{-3}$ ), Il and I2 (intermediate, $P \sim 2 \times 10^{3} \mathrm{~K} \mathrm{~cm}^{-3}$ ), and $\mathrm{C} 1$ and $\mathrm{C} 2$ (clumpy, $P \sim 10^{4} \mathrm{~K} \mathrm{~cm}^{-3}$ ). These models thus capture the properties of both high surface brightness spirals as well as low surface brightness disks. For each model we calculate the $\mathrm{H}_{2}$ gas mass fraction as a function of radius, as well as the $\mathrm{CO}$ emissivity and mass averaged gas temperature.

\section{Results}

\subsection{Molecular Gas Fractions}

Figure 1 shows $\Sigma_{\mathrm{H}_{2}} / \Sigma_{\mathrm{HI}}$ as a function of radius for several characteristic models. Several trends are immediately obvious:

- At fixed metallicity and ISM structure, lower surface brightness models have higher molecular fractions (Figure 1a). Because the ISRF scales with surface brightness, the molecules in the low surface brightness models are less apt to be dissociated by the background ISRF.

- At fixed surface brightness and ISM density structure, models with lower metallicity have lower molecular hydrogen gas content (Figure 1b). This result is due to the fact that dust grains act as formation sites for molecules; lower metallicities mean fewer dust grains to drive molecule formation.

- At fixed surface brightness and metallicity, clumpier ISM models have higher molecular gas fractions (Figure 1c). In clumpy models, a larger mass fraction of the gas is found in denser cores, and are shielded from the background ISRF. Molecules in diffuse ISM models lack this shielding, and are more easily dissociated by the UV background. 


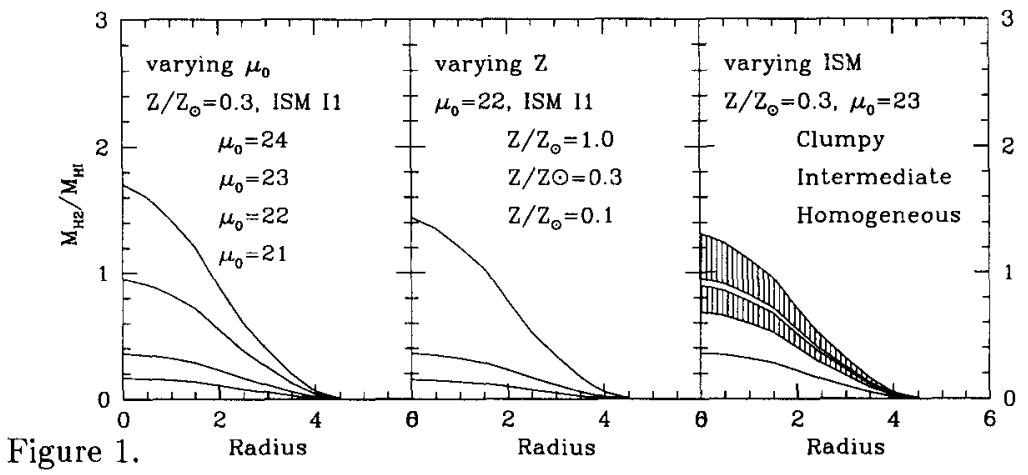

How well do these models describe actual disk galaxies? One point of constraint is provided by the Milky Way model. The high surface brightness, solar metallicity, and clumpy ISM model shows a mean $\mathrm{H}_{2} / \mathrm{HI}$ mass ratio $\sim 1$ averaged across the inner scale length of the disk, similar to that inferred for Milky Way-like Sb galaxies (Young \& Knezek 1989). This is not surprising, since the ISM models were scaled to the ISRF and structure of the Milky Way's ISM, but nonetheless it is reassuring that we recover the correct physical description for the given model inputs.

Assigning a model to LSB galaxies is not as straightforward. Certainly LSB disks are lower in metallicity (McGaugh 1992) and mean ISRF than the Milky Way. The density structure of the ISM in LSBs is not well determined, precisely due to the fact that $\mathrm{CO}$ measurements have not yielded any detections. Because of the lowered mass surface density of LSB disks (de Blok \& McGaugh 1996), it is likely that the ISM pressures are too low to support the amount of multi-phase structure found in the Milky Way. Models $\mathrm{H}$ (homogeneous) and I1 and I2 (intermediate) are therefore likely candidates to describe the density structure of LSB galaxies.

The models suggest that typical LSB galaxies have molecular contents which are only factors of $2-3$ below that of normal HSB spirals. The mass averaged gas temperatures in the molecular phase are by no means very cold, in contrast with their multi-phase counterparts (Mihos et al. 1998). Typical temperatures are around 30-50 K, similar to Spitzer-type HI clouds in our own Milky Way. Such high temperatures argue against efficient star formation, but self-consistent rates of the order of $\sim 0.05 \mathrm{M}_{\odot} \mathrm{yr}^{-1}$ appear feasible in these low metallicity environments (Norman \& Spaans 1997). In conclusion, The lack of detected CO emission in LSBs does not preclude the presence of modest amounts of molecular gas.

\section{2. $\mathrm{CO}$ Intensity and the $\mathrm{CO} / \mathrm{H}_{2}$ Conversion Factor}

To calculate the $\mathrm{CO}$ intensity of the models, the root mean square velocity of the interstellar clouds, the dispersion along the z-axis, is taken equal to 10 $\mathrm{km} \mathrm{s}^{-1}$, a typical value in the Milky Way and other galaxies. The turbulent velocity width of individual clouds is assumed equal to $3 \mathrm{~km} \mathrm{~s}^{-1}$. The integrated 


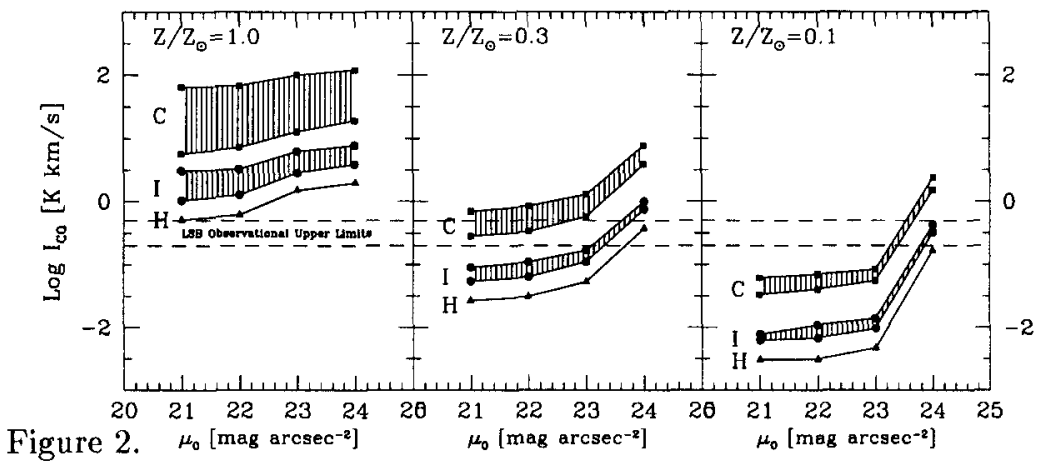

$\mathrm{CO}$ intensities are presented for face-on galaxies, integrated over the inner scale length. Figure 2 shows the variation in $\mathrm{I}(\mathrm{CO})$, the $\mathrm{CO} 1-0$ intensity in $\mathrm{K} \mathrm{km} \mathrm{s}^{-1}$, as a function of metallicity, surface brightness, and ISM structure. As with the $\mathrm{H}_{2}$ /HI mass ratio, several trends are immediately apparent: lower metallicity, higher surface brightnesses, and a more diffuse ISM all act to lower the CO intensity in the models. All these trends are as expected. Lower metallicities mean fewer carbon and oxygen atoms are available to form the $\mathrm{CO}$ molecule; higher surface brightnesses result in a stronger ISRF which destroys the CO molecule; and a diffuse ISM is less effective at shielding the $\mathrm{CO}$ molecules against radiative dissociation.

Also plotted on Figure 2 are the observational upper limits to the CO intensity of LSB galaxies determined by S90 and de Blok \& van der Hulst (1998). If LSBs have solar metallicity, these observations should have detected $\mathrm{CO}$ emission. But the subsolar metallicities of LSBs result in lowered $\mathrm{CO}$ intensities, making detection difficult. At $Z / Z_{\odot} \sim 0.3$, the $\mathrm{CO}$ emission is only a factor of $\sim 2-5$ below the observational limits, suggesting that deeper $\mathrm{CO}$ mapping may in fact reveal the molecular ISM of moderately metal poor LSBs. However, reducing the metallicity by another factor of three reduces the $\mathrm{CO}$ emission to levels 30 times fainter than the current observational limits; detecting these LSBs in CO will be very hard indeed.

Perhaps most germane to the observational status of molecular gas in LSB disk galaxies the the conversion factor $X=n\left(\mathrm{H}_{2}\right) / I(\mathrm{CO})$ (in units of $10^{21} \mathrm{~cm}^{-2}$ $\left.\left(\mathrm{K} \mathrm{km} \mathrm{s}^{-1}\right)^{-1}\right)$. Figure 3 shows this value calculated for the grid of ISM models. Far from being a constant value, $X$ shows significant and systematic variation between the different models. At solar metallicities, $X \sim 0.1-1$, spanning the "standard" value of X derived from Milky Way observations $(\sim 0.2-0.5$; see, e.g., Scoville \& Sanders 1987). Because the CO intensity scales non-linearly with density, $X$ has a strong dependence on the density structure of the ISM. Our models calculate the properties of the ISM over the inner disk scale length (3 $\mathrm{kpc}$ ), averaging over both cloud and inter-cloud regions. As the ISM becomes more clumpy, $\mathrm{X}$ decreases as the $\mathrm{CO}$ intensity rises faster than the $\mathrm{H}_{2}$ mass fraction. The value of $\mathrm{X}$ determined in the Milky Way may therefore be quite different from that applicable to galaxies with a more homogeneous ISM. 


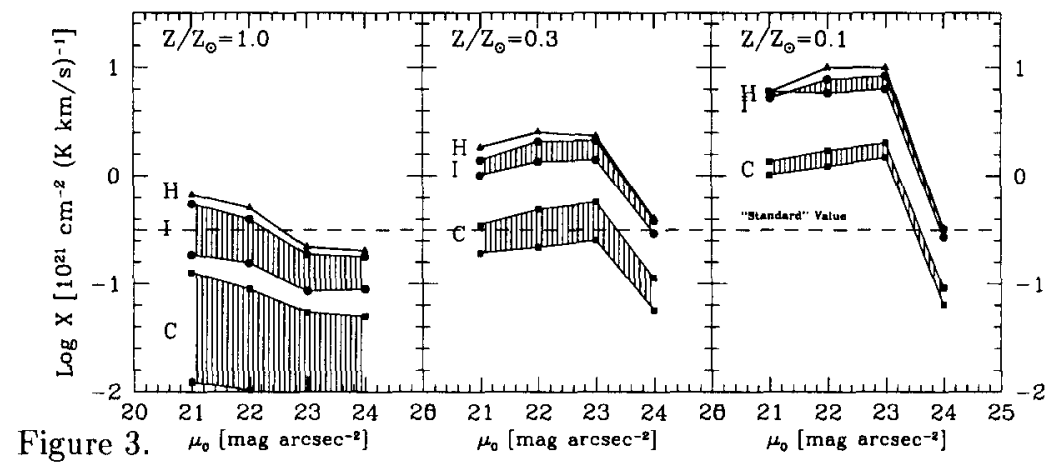

Aside from the dependence on ISM density structure, there is also a clear correlation between $\mathrm{X}$ and metallicity: as metallicity drops, the value of $\mathrm{X}$ increases. Such a trend has also been seen in observational data (e.g., Wilson 1995; Israel 1997), and in models of low metallicity clouds (Maloney \& Black 1988). The strength of this trend is still quite uncertain; Israel (1997) finds a strong dependence $(\partial \log X / \partial \log Z=-2.7 \pm 0.3)$, whereas Wilson (1995) derives a weaker relationship, $\partial \log X / \partial \log Z=-0.67 \pm 0.1$. In our models, the relationship is dependent on the ISM phase structure, but falls in the range $\partial \log X / \partial \log Z=-1$ to -2 .

Given the strong dependence on metallicity and ISM density structure, it is clear that use of the standard Milky Way value of X is suspect in LSB galaxies. Instead, we can now turn the problem around and ask, given our theoretical calculation of $\mathrm{X}$, what are the inferred constraints on the molecular gas fraction of LSBs from the CO studies of S90 and $\mathrm{dBvdH}$. If our models are correct, the upper limits on LSB molecular gas are an order of magnitude higher than inferred by these studies due to their use of the standard (low) value of X. Unfortunately, these upper limits then become rather weak: $M_{\mathrm{H}_{2}} / M_{\mathrm{HI}}<1$. While ruling out molecular gas as a dominant component of "dark matter" in LSB disks, these limits still allow for significant molecular gas fractions. More stringent limits on the molecular content of LSBs must await deeper CO observations.

\section{Discussion}

Our models indicate that even very low surface brightness galaxies may not be completely void of molecular gas - instead, 10-20\% of the ISM may be in molecular form. The physical conditions in this gas may be very different from the conditions in the molecular ISM of the Milky Way. If the ISM pressure is extremely low, as might be expected due to the low surface mass density of LSB disks, the molecular phase of the ISM will be diffuse and generally warmer than found in Galactic GMCs. These models also shed light on the lowered efficiency of star formation in LSB disks. Compared to HSBs, LSB galaxies have a lower fraction of molecular material from which they can produce stars. In addition, whatever molecular gas exists, it is in a more diffuse, probably warmer state than 
is typical for molecular material in HSBs. These warm temperatures and low densities act to help stabilize any existing molecular clouds against gravitational collapse.

The different evolutionary histories of HSB and LSB galaxies can be traced to differences in their disk surface densities and in the conditions of their ISMs. A plausible evolutionary scenario for HSB galaxies has been outlined by Norman \& Spaans (1997). In this scenario, once the proto-HSB gas disk forms, star formation begins at a retarded rate in the primordial molecular hydrogen ISM. This star formation generates supernovae and enriches the ISM, leading to a "phase transition" which leads to a multi-phase ISM that is able to cool efficiently and form stars efficiently, leading to a present-day HSB disk galaxy. In contrast, when a proto-LSB forms, it, too, forms a molecular ISM, but with a smaller molecular mass fraction and at lower surface density. At these low surface densities, it is difficult to trigger star formation or form/maintain a multi-phase ISM. As a result, the LSB evolves little from its primordial conditions, maintaining its low surface brightness and metallicity, and high gas fraction.

Acknowledgments. This work was supported by NASA through HF grant HF-01101.01-97A awarded by the STSCi.

\section{References}

Bothun, G.D., Impey, C., \& McGaugh, S.S. 1997, PASP, 109, 745

de Blok, W. J. G., \& McGaugh, S.S. 1996, ApJ, 469, L89

de Blok, W.J.G., \& van der Hulst, J.M. 1998, A\&A, submitted

de Blok, W.J.G., McGaugh, S.S., \& van der Hulst, J.M. 1996, MNRAS, 283, 18

Draine, B.T. 1978, ApJS, 36, 595

Field, G.B., Goldsmith, D., \& Habing, H.H. 1969, ApJ, 155, L149

Freeman, K.C. 1970, ApJ, 160, 811

Israel, F.P. 1997, A\&A, 328, 471

Maloney, P., \& Black, J.H. 1988, ApJ, 325, 389

McGaugh, S.S. 1992, Ph.D. thesis, University of Michigan

McGaugh, S.S., \& de Blok, W.J.G. 1997, ApJ, 481, 689

Mihos, J.C., Spaans, M., \& McGaugh, S.S. 1998, ApJ submitted

Norman, C.A., \& Spaans, M. 1997, ApJ, 480, 145

Scoville, N.Z., \& Sanders, D.B. 1987, in Interstellar Processes, eds. D.J. Hollenbach \& H.A. Thronson (Dordrecht: Reidel), 21

Schombert, J.S., Bothun, G.D., Impey, C.D., \& Mundy, L.G. 1990, AJ, 100, 1523 (S90)

Spaans, M. 1996, A\&A, 307, 271

van der Hulst, J.M., Skillman, E.D., Smith, T.R., Bothun, G.D., McGaugh, S.S. \& de Blok, W.J.G. 1993, AJ, 106, 548

Wilson, C.D. 1995, ApJ, 448, L97

Young, J.S., \& Knezek, P.M. 1989, ApJ, 347, L55 